\title{
How to manage the IVF during COVID-19 pandemic among diabetic females: a scientific perspective
}

\section{ABSTRACT}

Diabetes is a challenging clinical problem facing obstetricians and gynecologists when it comes to in vitro fertilization and embryo transfer (IVF-ET). During the COVID-19 pandemic we are living nowadays, COVID-19 becomes a new superimposing challenge for diabetic females need IVF-ET procedures.

The persistent lock-down of diabetic health facilities already advised by numerous organizations and contributing to challenging diabetes treatment is harmful to the whole population and in particular to patients with infertilities. Around $0.3 \%$ of all babies born last year were conceived with IVF-ET therapies worldwide. We recommend remedies to foresee more delicate infertility cases so as to prepare for a resumption of temporarily suspended fertility treatment.. In an age of crucial challenges for our national health services, complication prevention and tension management can help competent agencies and health providers identify patients that should be preferred to begin fertility treatment in a healthy environment.

What we consider as a possible possibility is the gradual restart of IVF, which needs many measures for diabetic patients. The problem of restarting IVF

Address for correspondence:

Ahmed M. El-Malky

King Saud University Medical City

King Khalid University Hospital

College of Medicine

Riyadh, Saudi Arabia

e-mail: aelmalky@ksu.edu.sa

Clinical Diabetology 2020, 9; 6: 372-377

DOI: $10.5603 /$ DK.2020.0067

Received: 20.11 .2020

Accepted: 29.11 .2020 installations after the current lockdown is real since each nation follows a certain recovery curve. Especially as a result of silent dissemination, attention should be provided to COVID-19 infection among patients and health-care staff after the restart of IVF therapy. (Clin Diabetol 2020; 9; 6: 372-377)

Key words: IVF, COVID-19, pandemic, diabetic, females

\section{Introduction}

Since the early stages of the pandemic, diabetic patients have been at the frontline, as rising epidemiological evidence showed that they are at higher risk of serious clinical effects of COVID-19 [1]. However, the present global condition does not discourage women from pursuing an interest in building a family and dreaming of a child. Even if she is diabetic and tries IVF, there is a possible danger of in-vitro fertilization (IVF), which may end in respiratory and cardiovascular problems, termed a severe ovarian hyper-stimulation syndrome [2]. Since COVID-19 may also induce respiratory and cardiovascular complications, it is unclear how women with COVID-19 manage a serious ovarian hyper-stimulation syndrome. There are no records of these problems at the moment [3].

The risk of serious ovarian hyper-stimulation syndrome may be increased by diabetes with or without other concomitant autoimmune diseases [4, 5]. For thorough guidance before embarking on ovarian stimulation, knowledge of the association of current conditions and the potential for vascular leakage disorders is required. In this demographic, prevention steps may be essential factors [5]. 
It was also established that pregnancy accomplished by IVF was linked, according to a study team in Greece, with a higher risk of gestational diabetes. These methods are considered to be correlated with a greater risk of pregnancy and birth complications. More than half a million babies are projected to be born worldwide through IVF [6].

However, fertility and IVF problems allow the patient to slip into the difficulty of finding the right period for IVF after the pandemic. Either delay or resume the extraction of IVF eggs and embryos, or freeze the eggs/embryos until date of expiry of eggs/ /embryos [7].

There is currently little scientific data on the connection between coronavirus and fertility [8]. We know that diseases will sometimes contribute to fever that can influence the treatment of infertility [9]. One research has shown that fever was correlated with a reduced amount of embryos, a longer duration and a higher amount of treatment needed during their freezing or IVF cycle. However there is no proof that the impact of fever on female fertility persists longer [10].

\section{COVID-19 and diabetes}

\section{Outcome of COVID-19 in diabetics}

People with COVID-19 diabetes are more likely to have poor prognosis and mortality. Given the high worldwide incidence of diabetes, the COVID-19 demographic represents a significant susceptible group [11]. The worse prognosis of persons with diabetes is likely to be a result of the syndromic aspect of the condition: hyperglycemia, older age, comorbidity and in particular, hypertension, obesity and cardiovascular disease - all lead to growing incidence in these individuals [12]. The scenario, however, is more complicated as it involves factoring in social variables such as inequality and race, as well as factors that become important at a time when a patient with extreme COVID-19 needs to be handled. Here the specialist must not only provide treatment for the health condition of the individual with diabetes, but must also closely match glucose-lowering medications with complex virus infection treatments [13].

\section{Diabetes management in COVID-19 patients}

Once again, diabetes treatment in patients with COVID-19 represents a significant clinical challenge, one that needs a well-integrated team solution, since this is an indispensable technique to minimize the likelihood of serious problems and mortality as far as possible [14]. Careful consideration of the multiple components that lead to poor prognosis of COVID-19 in patients with diabetes might be one of the most, if not the only, way to address the current condition and make it easier for our health services to be able to meet any potential problems in a timely and successful manner [15].

\section{Hidden relation between diabetes and COVID}

Finally, the inter-relationship between diabetes and COVID-19 needs further studies in order to explain the degree to which the particular mechanisms of the virus (e.g. its pancreatic $\beta$-cell tropism) could contribute to the deterioration of glycemic regulation and in certain situations, to the striking development of diabetic ketoacidosis or hyperglycemic hyperosmolar syndrome [16].

\section{Glycemic control and its impact}

Medical teams can maintain sufficient glycemic regulation in diabetic patients with COVID-19. This includes analysis of all possible effects that COVID-19 therapy may have had in patients with diabetes [17].

Treatment with chloroquine or hydroxychloroquine can induce hypoglycemia, especially in patients on insulin or sulfonylurea, due to their effects on insulin secretion, degradation and action [18]. Contrarily, medications such as lopinavir and ritonavir can contribute to hyperglycemia and exacerbate glycemic regulation [19]. These agents may induce hepatic and muscular toxicity, so vigilance is advised when used in conjunction with statins and in patients with fatty liver disease [20]. Pharmacokinetic reactions with antidiabetic drugs are often normal, triggering over-exposure or underexposure to either antiviral or anti-diabetic drugs [21]. Glucocorticoids have been used as symptomatic and anti-inflammatory therapy in individuals with COVID-19 with extreme acute respiratory distress syndrome. However, their usage can exacerbate insulin resistance, retain gluconeogenesis, worsen glycemic regulation, and trigger pronounced hyperglycemia. Glucocorticoids are known to impose their hyperglycemic impact by lowering insulin sensitivity and insulin release, and also by interaction with GLP-1 effects, and by increasing the development of glucagon [22].

\section{Diabetes and IVF}

How diabetes can impact woman fertility

Women who have diabetes are usually at a higher risk for conceiving complications. There are several causes that may lead to lower fertility rates: obesity, underweight, diabetes problems, PCOS (polycystic ovarian syndrome) or autoimmune disorder. The above factors are consistent with diabetes in women, which usually contributes to lowered fertility rates [23]. 
Patients with PCOS are candidates for IVF

Polycystic ovary syndrome - PCOS is a medical disorder in which a large number of cysts form on the ovary and it may influence fertility due to irregular or missing cycles. PCOS is primarily linked with type 2 diabetes and obesity [24]. Cycles are considered irregular if periods occur at intervals of 35 days or more. Periods are considered to be missing when a woman who has previously had regular cycles is missing a period for 6 months or more [25].

\section{Premature menopause and diabetes}

Premature menopause - frequently correlated with type 1 diabetes, premature menopause occurs when a woman stops having periods before the age of 40. Endometrial cancer (uterine cancer). This condition is more frequent in women with type 2 diabetes and PCOS, and can contribute to infertility if not treated early [26]. Microvascular and cardiovascular risks - findings indicate that people with type 1 diabetes who have microvascular or cardiovascular complications have significantly lower fertility rates [27].

\section{The new triad (IVF, OSS and diabetes)}

Patients receiving gonadotrophin ovary stimulation for IVF cycles are vulnerable to Ovarian Stimulation Syndrome (OSS). Presence of unregulated diabetes is a risk factor that raises the likelihood of ovarian hyper-stimulation; as shown in several trials, metformin has had a beneficial impact on the prevention of such complications among diabetic females pursuing IVF pregnancy. Reduction of estradiol amounts on the day of administration of HCG. Another potential reason is that the impact of berberine or metformin on the reduction of androgen and insulin levels may lead to reduced estradiol concentrations [28-31].

Data also suggests that metformin has a clear impact on human ovarian steroidogenesis [32]. In addition, metformin has also been shown to directly suppress the activity of aromatase enzyme [29]. These findings may also explain the rapid biochemical improvements in serum testosterone and estradiol concentrations observed in our study after a short course of berberine or metformin.

Safety is a key consideration for women undergoing IVF. Hyperinsulinemia is a risk factor for OHSS, as women with PCOS who are hyperinsulinemic have a higher level of E2 and a greater incidence of ovarian hyper-stimulation during ovarian stimulation with $\mathrm{FSH}$, compared to those with normoinsulinemia [29].

\section{IVF and gestational diabetes}

Pregnant mothers who used in vitro fertilization to conceive are $53 \%$ more likely to experience gestational diabetes than women who conceived spontaneously, according to research results provided at the annual meeting of the European Association for the Study of Diabetes [33].

"These results underscore the significance of early diagnosis of gestational diabetes in pregnant women with assisted reproductive technologies that may contribute to an increase in lifestyle activity from the previous to the IVF era and the first trimester of pregnancy, especially in high-risk communities, in order to reduce the risk of gestational diabetes."

In a systematic study and meta-analysis, Anagnostis at al. [33] reviewed evidence from 17 matched and 21 unmatched case-control trials conducted between 1995 and 2017, contrasting the probability of gestational diabetes in singleton pregnancy with assisted reproductive technologies (IVF and intracytoplasmic sperm injection) vs. random conception ( $n=1,893,599)$ [34]. Studies were removed if conception was accomplished by ovulation induction or intrauterine insemination. Researchers used maternal age, parity and ethnicity to balance aided reproductive groups and spontaneous reproduction groups.

Across trials, 4,766 out of 63,760 people who experienced assisted reproduction and 158,526 out of $1,870,734$ women who became pregnant naturally acquired gestational diabetes [35]. "This thorough review of the best available data to date indicates that singleton pregnancy obtained by IVF is correlated with an elevated risk of developing gestational diabetes relative to pregnancies spontaneously born." Anagnostis said in a press release. "The precise cause remains unknown, and whether this risk is related to medical action or to the inherent infertility status of couples undergoing assisted reproduction is not yet well known and needs more study."

\section{COVID-19 and IVF}

\section{COVID-19 impact on fertility clinics}

Since most coronavirus cases are related to travel in places with ongoing outbreaks, fertility clinics are advising patients to postpone IVF or other fertility care. Previously, it was proposed by the Society for Assisted Reproductive Technology (SART) and the American Society for Reproductive Medicine (ASRM) that patients with a high risk of coronavirus (current symptoms, verified patient infection within 14 days of initiation of symptoms, or a positive coronavirus test result) should aim to prevent pregnancy [36]. 
These recommendations apply to procedures in egg or sperm donors and surrogate mothers, or IVF transfers. In context, if signs of respiratory failure (common with coronavirus) occur, anesthesia used during the extraction period can further inhibit respiration while the immune system may still be weakened. Going ahead with a transfer period may pose a danger to the applicant, clinic personnel, or other patients, and is usually really not the right option [37].

At the beginning of April, ASRM had initially suggested halting the implementation of new therapy periods, including activation of ovulation, IUIs, IVF retrievals and exchanges, and non-urgent cryopreservation of gametes owing to coronavirus pandemics. Patients and physicians were actively advised to postpone any transfers of embryos, fresh or frozen, and allowed to collaborate closely to decide how "urgent" the patient's treatment could be [38].

In other terms, whether fertility treatment was super time-sensitive (say, in the case of age or reduced ovarian reserve) and a protracted pause may have an effect on the result that might have been deemed urgent. The ASRM suggested the cessation of non-timesensitive activities, reducing in-person experiences and promoting the usage of telehealth. However, patients that were mid-cycle would have been willing to pursue their care.

\section{COVID-19 impact on fertility}

There is currently insufficient clinical information on the relationship between coronavirus and fertility. But this is a fairly touch-and-go scenario. COVID-19 is relatively recent, so the $\mathrm{CDC}$ and other scientists would need to continue tracking its impact on fertility and beyond [39].

What we do know is that viruses will often trigger a fever that can affect fertility care. One research indicated that fever during the egg freeze or IVF period was correlated with a lower number of eggs recovered, a longer cycle, and a higher amount of medication needed. There is no proof, however, that fever causes longer-term effects on female fertility [40].

\section{COVID-19 impact on pregnancy}

We know that full-term babies born from women with active COVID-19 infections have performed well while serious illness (COVID-19 or otherwise) can contribute to premature labor. A very small case-analysis of COVID-19-affected women who delivered by C-section found that the virus had not been transmitted via amniotic fluid, cord blood or breastmilk, although it is still uncertain if transmission is feasible. However, in another review, preeclampsia was identified in 6 of 8 women with serious COVID-19 pneumonia admitted to ICU, although no preeclampsia symptoms were found in 34 participants with more moderate coronavirus [41].

However these details are somewhat insufficient. Abortion and stillbirth, has been shown in cases of infection with other associated coronaviruses (SARS-CoV and MERS-CoV) during pregnancy, and the CDC states that high fever during the first trimester of pregnancy may raise the risk of some birth defects. This describes the prudent attitude of ASRM to begin transfer cycles at this period, although there is no evidence yet on the effect of COVID-19 on the fetus during the first or second trimesters of pregnancy [42].

\section{Conclusion}

To our knowledge the inter-relationship among those three elements of the triad, "Diabetes, COVID and Fertility", was not addressed in literature. However, it was noticed obviously in many cases managed particularly in IVF clinics that there was a hidden relation among those three factors. Diabetic patients who attended our clinics for IVF trials during the COVID-19 pandemic experienced unexpected procedure failures and outcomes. This hypothesis obliged us to start our question by a review of literature to find that this topic was not discussed before. We recommend further researches in this new topic to find a causative and any associated risk factors.

\section{Conflict of interest}

The authors declare that there is no conflict of interest.

\section{REFERENCES}

1. Abdi A, Jalilian M, Sarbarzeh PA, et al. Diabetes and COVID-19: A systematic review on the current evidences. Diabetes Res Clin Pract. 2020; 166: 108347, doi: 10.1016/j.diabres.2020.108347, indexed in Pubmed: 32711003.

2. Tandulwadkar SR, Lodha PA, Mangeshikar NT. Obstetric complications in women with IVF conceived pregnancies and polycystic ovarian syndrome. J Hum Reprod Sci. 2014; 7(1): 13-18, doi: 10.4103/0974-1208.130802, indexed in Pubmed: 24829525.

3. Fabregues F, Peñarrubia J. Assisted reproduction and thromboembolic risk in the COVID-19 pandemic. Reprod Biomed Online. 2020; 41(3): 361-364, doi: 10.1016/j.rbmo.2020.06.013, indexed in Pubmed: 32660814.

4. Selter J, Wen T, Palmerola KL, et al. Life-threatening complications among women with severe ovarian hyperstimulation syndrome. Am J Obstet Gynecol. 2019; 220(6): 575.e1-575.e11, doi: 10.1016/j.ajog.2019.02.009, indexed in Pubmed: 30742828.

5. Kilpatrick CR, Ratts VS, Simckes E, et al. Severe ovarian hyperstimulation syndrome in patients with autoimmune disorders: a report of two cases. J Reprod Med. 2014; 59(11-12): 591-595, indexed in Pubmed: 25552133.

6. Kouhkan A, Khamseh ME, Pirjani R, et al. Obstetric and perinatal outcomes of singleton pregnancies conceived via assisted reproductive technology complicated by gestational diabetes 
mellitus: a prospective cohort study. BMC Pregnancy Childbirth. 2018; 18(1): 495, doi: 10.1186/s12884-018-2115-4, indexed in Pubmed: 30547777

7. Szymanska M, Horosz E, Szymusik I, et al. Gestational diabetes in IVF and spontaneous pregnancies. Neuro Endocrinol Lett. 2011; 32(6): 885-888, indexed in Pubmed: 22286793.

8. Segars J, Katler Q, McQueen DB, et al. American Society for Reproductive Medicine Coronavirus/COVID-19 Task Force. Prior and novel coronaviruses, Coronavirus Disease 2019 (COVID-19), and human reproduction: what is known? Fertil Steril. 2020; 113(6): 1140-1149, doi: 10.1016/j.fertnstert.2020.04.025, indexed in Pubmed: 32482250

9. Diriba K, Awulachew E, Getu E. The effect of coronavirus infection (SARS-CoV-2, MERS-CoV, and SARS-CoV) during pregnancy and the possibility of vertical maternal-fetal transmission: a systematic review and meta-analysis. Eur J Med Res. 2020; 25(1): 39, doi: 10.1186/s40001-020-00439-w, indexed in Pubmed: 32887660.

10. Anifandis G, Messini CI, Daponte A, et al. COVID-19 and fertility: a virtual reality. Reprod Biomed Online. 2020; 41(2): 157-159, doi: 10.1016/j.rbmo.2020.05.001, indexed in Pubmed: 32466995.

11. Zhu L, She ZG, Cheng Xu, et al. Association of Blood Glucose Control and Outcomes in Patients with COVID-19 and Pre-existing Type 2 Diabetes. Cell Metab. 2020; 31(6): 1068-1077.e3, doi: 10.1016/j.cmet.2020.04.021, indexed in Pubmed: 32369736.

12. Huang I, Lim MA, Pranata R. Diabetes mellitus is associated with increased mortality and severity of disease in COVID-19 pneumonia - A systematic review, meta-analysis, and meta-regression. Diabetes Metab Syndr. 2020; 14(4): 395-403, doi: 10.1016/j. dsx.2020.04.018, indexed in Pubmed: 32334395.

13. Tadic M, Cuspidi C, Sala C. COVID-19 and diabetes: Is there enough evidence? J Clin Hypertens (Greenwich). 2020; 22(6): 943-948, doi: 10.1111/jch.13912, indexed in Pubmed: 32472662.

14. Pugliese $G$, Vitale $M$, Resi V, et al. Is diabetes mellitus a risk factor for COronaVIrus Disease 19 (COVID-19)? Acta Diabetol. 2020; 57(11): 1275-1285, doi: 10.1007/s00592-020-01586-6, indexed in Pubmed: 32865671.

15. Rahimi L, Malek M, Ismail-Beigi F, et al. Challenging issues in the management of cardiovascular risk factors in diabetes during the COVID-19 pandemic: a review of current literature. Adv Ther. 2020; 37(8): 3450-3462, doi: 10.1007/s12325-020-01417-8, indexed in Pubmed: 32632851.

16. Hussain A, Bhowmik B, do Vale Moreira NC. COVID-19 and diabetes: Knowledge in progress. Diabetes Res Clin Pract. 2020; 162: 108142, doi: 10.1016/j.diabres.2020.108142, indexed in Pubmed: 32278764.

17. Singh AK, Gupta R, Ghosh A, et al. Diabetes in COVID-19: Prevalence, pathophysiology, prognosis and practical considerations. Diabetes Metab Syndr. 2020; 14(4): 303-310, doi: 10.1016/j. dsx.2020.04.004, indexed in Pubmed: 32298981.

18. Unübol M, Ayhan M, Guney E. Hypoglycemia induced by hydroxychloroquine in a patient treated for rheumatoid arthritis. J Clin Rheumatol. 2011; 17(1): 46-47, doi: 10.1097/ RHU.0b013e3182098e1f, indexed in Pubmed: 21169846.

19. Paengsai N, Jourdain G, Salvadori N, et al. Recommended firstline antiretroviral therapy regimens and risk of diabetes mellitus in HIV-infected adults in resource-limited settings. Open Forum Infect Dis. 2019; 6(10): ofz298, doi: 10.1093/ofid/ofz298, indexed in Pubmed: 31660327.

20. Bruno R, Sacchi P, Maiocchi L, et al. Hepatotoxicity and antiretroviral therapy with protease inhibitors: A review. Dig Liver Dis. 2006; 38(6): 363-373, doi: 10.1016/j.dld.2006.01.020, indexed in Pubmed: 16631422.

21. Liverpool COVID-19 interactions. https://www.covid19-druginteractions.org/ (5.05.2020).

22. Isidori AM, Arnaldi G, Boscaro M, et al. COVID-19 infection and glucocorticoids: update from the Italian Society of Endocrinology Expert Opinion on steroid replacement in adrenal insufficiency.
J Endocrinol Invest. 2020; 43(8): 1141-1147, doi: 10.1007/ s40618-020-01266-w, indexed in Pubmed: 32335855.

23. Thong E, Codner E, Laven J, et al. Diabetes: a metabolic and reproductive disorder in women. The Lancet Diabetes \& Endocrinology. 2020; 8(2): 134-149, doi: 10.1016/s2213-8587(19)30345-6.

24. Swanton A, Storey L, McVeigh E, et al. IVF outcome in women with PCOS, PCO and normal ovarian morphology. Eur J Obstet Gynecol Reprod Biol. 2010; 149(1): 68-71, doi: 10.1016/j. ejogrb.2009.11.017, indexed in Pubmed: 20022685.

25. Li HW, Lee VC, Lau EY, et al. Cumulative live-birth rate in women with polycystic ovary syndrome or isolated polycystic ovaries undergoing in-vitro fertilisation treatment. J Assist Reprod Genet. 2014; 31(2): 205-211, doi: 10.1007/s10815-013-0151-6, indexed in Pubmed: 24337962.

26. Anagnostis $\mathrm{P}$, Christou K, Artzouchaltzi AM, et al. Early menopause and premature ovarian insufficiency are associated with increased risk of type 2 diabetes: a systematic review and meta-analysis. Eur J Endocrinol. 2019; 180(1): 41-50, doi: 10.1530/EJE-18-0602, indexed in Pubmed: 30400047

27. Paschou SA, Papanas N. Type 2 Diabetes Mellitus and Menopausal Hormone Therapy: An Update. Diabetes Ther. 2019; 10(6): 2313-2320, doi: 10.1007/s13300-019-00695-y, indexed in Pubmed: 31549295.

28. Tang $\mathrm{T}$, Glanville J, Orsi $\mathrm{N}$, et al. The use of metformin for women with PCOS undergoing IVF treatment. Hum Reprod. 2006; 21(6): 1416-1425, doi: 10.1093/humrep/del025, indexed in Pubmed: 16501038

29. Haas DA, Carr BR, Attia GR. Effects of metformin on body mass index, menstrual cyclicity, and ovulation induction in women with polycystic ovary syndrome. Fertil Steril. 2003; 79(3): 469-481, doi: 10.1016/s0015-0282(02)04800-8, indexed in Pubmed: 12620424.

30. Harborne L, Fleming $\mathrm{R}$, Lyall $\mathrm{H}$, et al. Descriptive review of the evidence for the use of metformin in polycystic ovary syndrome. Lancet. 2003; 361(9372): 1894-1901, doi: 10.1016/501406736(03)13493-9, indexed in Pubmed: 12788588.

31. Kjøtrød SB, von Düring V, Carlsen SM. Metformin treatment before IVF/ICSI in women with polycystic ovary syndrome; a prospective, randomized, double blind study. Hum Reprod. 2004; 19(6): 1315-1322, doi: 10.1093/humrep/deh248, indexed in Pubmed: 15117902.

32. Mansfield R, Galea R, Brincat M, et al. Metformin has direct effects on human ovarian steroidogenesis. Fertil Steril. 2003; 79(4): 956-962, doi: 10.1016/s0015-0282(02)04925-7, indexed in Pubmed: 12749437.

33. Anagnostis $P$, et al. Abstract 921. Presented at: European Association for the Study of Diabetes Annual Meeting; Sept. 16-20, 2019; Barcelona, Spain.

34. Maroufizadeh S, Navid B, Alizadeh A, et al. Risk of gestational diabetes mellitus following assisted reproductive technology: systematic review and meta-analysis of 59 cohort studies. J Matern Fetal Neonatal Med. 2019 [Epub ahead of print]: 1-10, doi: 10.1080/14767058.2019.1670790, indexed in Pubmed: 31570010

35. IVF, Assisted Reproduction Techiques Linked to Increased Gestational Diabetes. (n.d.). https://www.hcplive.com/view/ivf-assistedreproduction-techiques-linked-to-increased-gestational-diabetes (18.12.2020)

36. Requena A, Cruz M, Vergara V, et al. A picture of the covid-19 impact on IVIRMA fertility treatment clinics in Spain and Italy. Reprod Biomed Online. 2020; 41(1): 1-5, doi: 10.1016/j. rbmo.2020.04.015, indexed in Pubmed: 32451301.

37. Monteleone PAa, Nakano M, Lazar V, et al. A review of initial data on pregnancy during the COVID-19 outbreak: implications for assisted reproductive treatments. JBRA Assist Reprod. 2020; 24(2): 219-225, doi: 10.5935/1518-0557.20200030, indexed in Pubmed: 32301600 . 
38. Peyronnet V, Sibiude J, Deruelle P, et al. [SARS-CoV-2 infection during pregnancy. Information and proposal of management care. CNGOF]. Gynecol Obstet Fertil Senol. 2020; 48(5): 436-443, doi: 10.1016/j.gofs.2020.03.014, indexed in Pubmed: 32199996.

39. Blumenfeld Z. Possible impact of COVID-19 on fertility and assisted reproductive technologies. Fertil Steril. 2020; 114(1): 56-57, doi: 10.1016/j.fertnstert.2020.05.023, indexed in Pubmed: 32622412.

40. Zhu C, Wu J, Liang Y, et al. Fertility intentions among couples in Shanghai under COVID-19: A cross-sectional study. Int J Gynaecol
Obstet. 2020 [Epub ahead of print], doi: 10.1002/ijgo.13366, indexed in Pubmed: 32880942.

41. Juan J, Gil MM, Rong Z, et al. Effect of coronavirus disease 2019 (COVID-19) on maternal, perinatal and neonatal outcome: systematic review. Ultrasound Obstet Gynecol. 2020; 56(1): 15-27. doi: 10.1002/uog.22088, indexed in Pubmed: 32430957.

42. Huntley BJF, Huntley ES, Di Mascio D, et al. Rates of Maternal and Perinatal Mortality and Vertical Transmission in Pregnancies Complicated by Severe Acute Respiratory Syndrome Coronavirus 2 (SARS-Co-V-2) Infection: A Systematic Review. Obstet Gynecol. 2020; 136(2): 303-312, doi: 10.1097/AOG.0000000000004010, indexed in Pubmed: 32516273. 